\title{
STIM and Orai1 Variants in Store-Operated Calcium Entry
}

\author{
Juan A. Rosado ${ }^{*}$, Raquel Diez ${ }^{1}$, Tarik Smani ${ }^{2}$ and Isaac Jardín ${ }^{1}$ \\ ${ }^{1}$ Department of Physiology, Cell Physiology Research Group, University of Extremadura, Cáceres, Spain, ${ }^{2}$ Department of \\ Medical Physiology and Biophysic, Institute of Biomedicine of Seville, Virgen del Rocio University Hospital, Consejo Superior \\ de Investigaciones Cientificas, University of Seville, Sevilla, Spain
}

\section{OPEN ACCESS}

Edited by: Antonio Ferrer-Montiel, Universitas Miguel Hernández, Spain

Reviewed by: Hiroshi Hibino,

Niigata University, Japan Carlos Villalobos,

Consejo Superior de Investigaciones Cientificas, Spain

*Correspondence:

Juan A. Rosado jarosado@unex.es

Specialty section: This article was submitted to Pharmacology of Ion Channels and Channelopathies, a section of the journal

Frontiers in Pharmacology

Received: 01 December 2015 Accepted: 30 December 2015

Published: 13 January 2016

Citation

Rosado JA, Diez R, Smani T and Jardín I (2016) STIM and Orai1 Variants in Store-Operated Calcium

Entry. Front. Pharmacol. 6:325. doi: 10.3389/fphar.2015.00325
Store-operated $\mathrm{Ca}^{2+}$ entry (SOCE) is an ubiquitous mechanism for $\mathrm{Ca}^{2+}$ entry in eukaryotic cells. This route for $\mathrm{Ca}^{2+}$ influx is regulated by the filling state of the intracellular $\mathrm{Ca}^{2+}$ stores communicated to the plasma membrane channels by the proteins of the Stromal Interaction Molecule (STIM) family, STIM1, and STIM2. Storedependent, STIM1-modulated, channels include the $\mathrm{Ca}^{2+}$ release-activated $\mathrm{Ca}^{2+}$ channels, comprised of subunits of Orai proteins, as well as the store-operated $\mathrm{Ca}^{2+}$ (SOC) channels, involving Orai1, and members of the canonical transient receptor potential family of proteins. Recent studies have revealed the expression of splice variants of STIM1, STIM2, and Orai1 in different cell types. While certain variants are ubiquitously expressed, others, such as STIM1L, show a more restricted expression. The splice variants for STIM and Orai1 proteins exhibit significant functional differences and reveal that alternative splicing enhance the functional diversity of STIM1, STIM2, and Orai1 genes to modulate the dynamics of $\mathrm{Ca}^{2+}$ signals.

Keywords: calcium entry, STIM1, STIM2, orai1, splice variants

\section{INTRODUCTION}

Eukaryotic cells finely modulate cytosolic calcium concentration $\left(\left[\mathrm{Ca}^{2+}\right]_{\mathrm{c}}\right)$ to trigger a myriad of physiological events, from short term responses, such muscle contraction, impulse transmission, secretion, and aggregation, to long term events, including activation of transcription factors, growth and in the last instance, apoptosis, and cellular death. Evolution has provided the cells with a highly complex machinery, which finely tunes, orchestrates and coordinates intracellular $\mathrm{Ca}^{2+}$ homeostasis. Physiological agonists modulates $\left[\mathrm{Ca}^{2+}\right]_{\mathrm{c}}$ by the activation of more or less selective $\mathrm{Ca}^{2+}$ channels and transporters, both in the intracellular $\mathrm{Ca}^{2+}$ compartments [endoplasmic reticulum (ER), mitochondria or acidic vesicles] and in the plasma membrane (PM). Once the stimulus ends, $\left[\mathrm{Ca}^{2+}\right]_{\mathrm{c}}$ returns to basal level and the cell is ready for a new stimulation (Berridge et al., 2003; Redondo and Rosado, 2015). Cells have a number of mechanisms to induce $\mathrm{Ca}^{2+}$ entry and although all these events were studied in the past as independent pathways, mostly due to technical challenges to address $\mathrm{Ca}^{2+}$ signal as a whole, new studies and a deeper comprehension of $\mathrm{Ca}^{2+}$ entry support a sophisticated relation encompassed by all these pathways (Mignen et al., 2007; Wang et al., 2010; Rodriguez-Moyano et al., 2013; Zhang et al., 2014).

Store-Operated Calcium Entry (SOCE), a major mechanism for $\mathrm{Ca}^{2+}$ influx, is regulated by the filling state of the intracellular $\mathrm{Ca}^{2+}$ reservoirs, mainly the ER. A reduction in the intraluminal $\mathrm{ER} \mathrm{Ca}^{2+}$ concentration $\left(\left[\mathrm{Ca}^{2+}\right]_{\mathrm{ER}}\right)$, evokes the opening of channels in the PM leading to $\mathrm{Ca}^{2+}$ entry from the extracellular medium (Putney, 1986). After intense investigation, the mechanism that communicate the filling state of the intracellular $\mathrm{Ca}^{2+}$ stores to the PM channels was found to 
be mediated by the Stromal Interaction Molecule 1 (STIM1), a protein discovered in Oritani and Kincade (1996) and known as a cell-cell interaction mediator. STIM1 is the ER $\mathrm{Ca}^{2+}$ sensor that stimulate $\mathrm{Ca}^{2+}$ entry, triggering the activation of store-operated channels located in the PM (Roos et al., 2005; Zhang et al., 2005). Concerning the $\mathrm{Ca}^{2+}$-permeable channels that conduct SOCE, soon after the identification of STIM1 as the ER $\mathrm{Ca}^{2+}$ sensor Orail was proposed as the pore-forming subunit of the $\mathrm{Ca}^{2+}$ release-activated $\mathrm{Ca}^{2+}$ (CRAC) channels (Feske et al., 2005, 2006; Mercer et al., 2006; Peinelt et al., 2006; Prakriya et al., 2006). In addition, STIM1 might activate the less $\mathrm{Ca}^{2+}$ selective store-operated $\mathrm{Ca}^{2+}$ (SOC) channels, which require the interaction of Orail with the canonical transient receptor potential (TRP) family member TRPC1 (Rosado and Sage, 2000; Singh et al., 2000; Huang et al., 2006; Yuan et al., 2007; Jardin et al., 2008; Cheng et al., 2011, 2013; Choi et al., 2014; Desai et al., 2015). STIM1 activates Orail through a cytosolic STIM1Orail activation region (SOAR; aa 344-442; Yuan et al., 2009) also identified as the CRAC activating domain (CAD; aa 342448; Park et al., 2009); the Orai-activating small fragment (OASF; aa 233-450/474; Muik et al., 2009) and the Ccb9 (aa 339-44; Kawasaki et al., 2009). SOAR dimerization is essential for the activation of Orail and the polybasic region (aa 382-387) within the SOAR structure is required for Orail binding (Yang et al., 2012). The activation of TRPC1 by STIM1 has been reported to require both, the SOAR region, which is important for the STIM1-TRPC1 interaction (Lee et al., 2014), and the last 14 amino acids of STIM1, which constitute a polybasic lysine-rich domain required for the activation of TRPC channels by STIM1 upon store depletion (Zeng et al., 2008).

Furthermore, Orai1, together with Orai3, and the PMresident STIM1 have also been reported to participate in a store-independent mechanism for $\mathrm{Ca}^{2+}$ entry activated by arachidonate (Mignen et al., 2008a,b, 2009), which reveals the diversity and complexity of the regulation of $\mathrm{Ca}^{2+}$ entry in eukaryotic cells.

\section{STIM PROTEINS}

Members of the STIM family, STIM1 (Figure 1) and STIM2 (Figure 2), have highly conserved structure and present slightly divergences giving them different functions. Upon store depletion, STIM1 oligomerizes and redistributes into discrete puncture nearby the PM (Luik et al., 2008; Cahalan, 2009; Park et al., 2009; Covington et al., 2010).

Both STIMs are single spanning transmembrane (TM) proteins that are located mainly in the ER (Roos et al., 2005; Zhang et al., 2005; Baba et al., 2006), but also in acidic stores (Zbidi et al., 2011) and in the PM (Sabbioni et al., 1999; Spassova et al., 2006; Jardin et al., 2013). The STIM N-terminal region is located in the intraluminal compartment (or the extracellular medium when located in the PM), harboring the canonical and hidden EF-hand (hEF) motives (for STIM1 aa 63-128; Liou et al., 2005; Roos et al., 2005). The $\mathrm{Ca}^{2+}$ binding canonical EF-hand is the $\mathrm{Ca}^{2+}$ sensor. Mutations within this region incapacitate $\mathrm{Ca}^{2+}$ association, thus, inducing constitutive $\mathrm{Ca}^{2+}$ entry (Spassova et al., 2006). Next, STIM presents the steril- $\alpha$-motif (SAM) domain (aa 132-200) exhibiting distinct properties in STIM1 and STIM2 (Stathopulos et al., 2009; Zheng et al., 2011). SAM is followed by the TM domain (aa 214-343), which has been recently shown to undergo structural changes from the resting state, where $\mathrm{Ca}^{2+}$ is bond to the EF-hand, to the activated one, when store depletion occurs (Ma et al., 2015). Located in the cytosol, STIM C-terminus comprises 3 conserved coiledcoil (CC) domains, CC1 (aa 238-343), CC2 (aa 363-389), and CC3 (aa 363-389), the CRAC modulatory domain (CMD; aa 448-530), which includes the STIM1 homomerization domain SHD (aa 420-450), followed by a serine/proline rich region (aa 600-629) and a lysine-rich region (aa 671-685) at the very end of the C-terminus, that binds to membrane phospholipids, thus anchoring STIM1 toward its target (Liou et al., 2005). Furthermore, the polybasic lysine-rich region regulates $\mathrm{Ca}^{2+}$ entry by PM-resident STIM1 (Jardin et al., 2009, 2013). The long CC1 domain might be separated into $\mathrm{CC}_{\alpha 1}, \mathrm{CC}_{\alpha 2}$, and $\mathrm{CC1}_{\alpha 3}$ (Soboloff et al., 2012; Yang et al., 2012; Stathopulos et al., 2013). Furthermore, CC2 and CC3 domains, which comprise the SOAR domain, could be divided in four regions, $\mathrm{S} \alpha 1, \mathrm{~S} \alpha 2$, S $\alpha 3$, and S $\alpha 4$ (Yang et al., 2012; Wang et al., 2014). Beside the differences between STIM1 and STIM2, the CC regions are highly conserved, presenting, however, noticeable disparity in their functions (Wang et al., 2014).

\section{ORAI PROTEINS}

The Orai channels family is composed by three remarkably conserved homologs: Orai1, Orai2, and Orai3 (Feske et al., 2005; Mercer et al., 2006; Zhang et al., 2006; Gwack et al., 2007; Rothberg et al., 2013). A single Orail monomer spams four times the PM, exposing two loops ( 1 and 3 ) to the extracellular medium and with the $\mathrm{N}$ - and $\mathrm{C}$-terminus domains and one loop (2) facing the cytoplasm (Figure 3). Both, N- and C-termini are required for STIM1 interaction and regulation (Muik et al., 2009; Park et al., 2009; Yuan et al., 2009; Derler et al., 2013; Palty et al., 2015; Palty and Isacoff, 2016).

Prior to its crystal structure determination in Drosophila, the human Orail channel was thought to form a tetramer with high selectivity to $\mathrm{Ca}^{2+}$ (Mignen et al., 2008b; Penna et al., 2008; Maruyama et al., 2009). Crystallization of Drosophila Orai1 showed a hexameric molecule permeable to $\mathrm{Ca}^{2+}$ as well as to monovalent ions in the presence of divalent cations (Hou et al., 2012; Thompson and Shuttleworth, 2013a). Furthermore, the Orai channels may form heteropentamers (3 Orail and 2 Orai3) to function as arachidonate-regulated $\mathrm{Ca}^{2+}$ (ARC) channels, a store-independent channel regulated by the STIM1 population located in the PM (Thompson and Shuttleworth, 2013b; Zhang et al., 2014).

Regarding CRAC channels, and despite that further studies are needed to determine human Orail structure, it is mostly accepted that each Orail channel comprises 6 Orail monomers, accurately arranged, forming the highly $\mathrm{Ca}^{2+}$ selective ion channel in the PM. The pore is located amid the hexamer, involving the six TM1 domains and including the residues 74-90 (ETON region) 


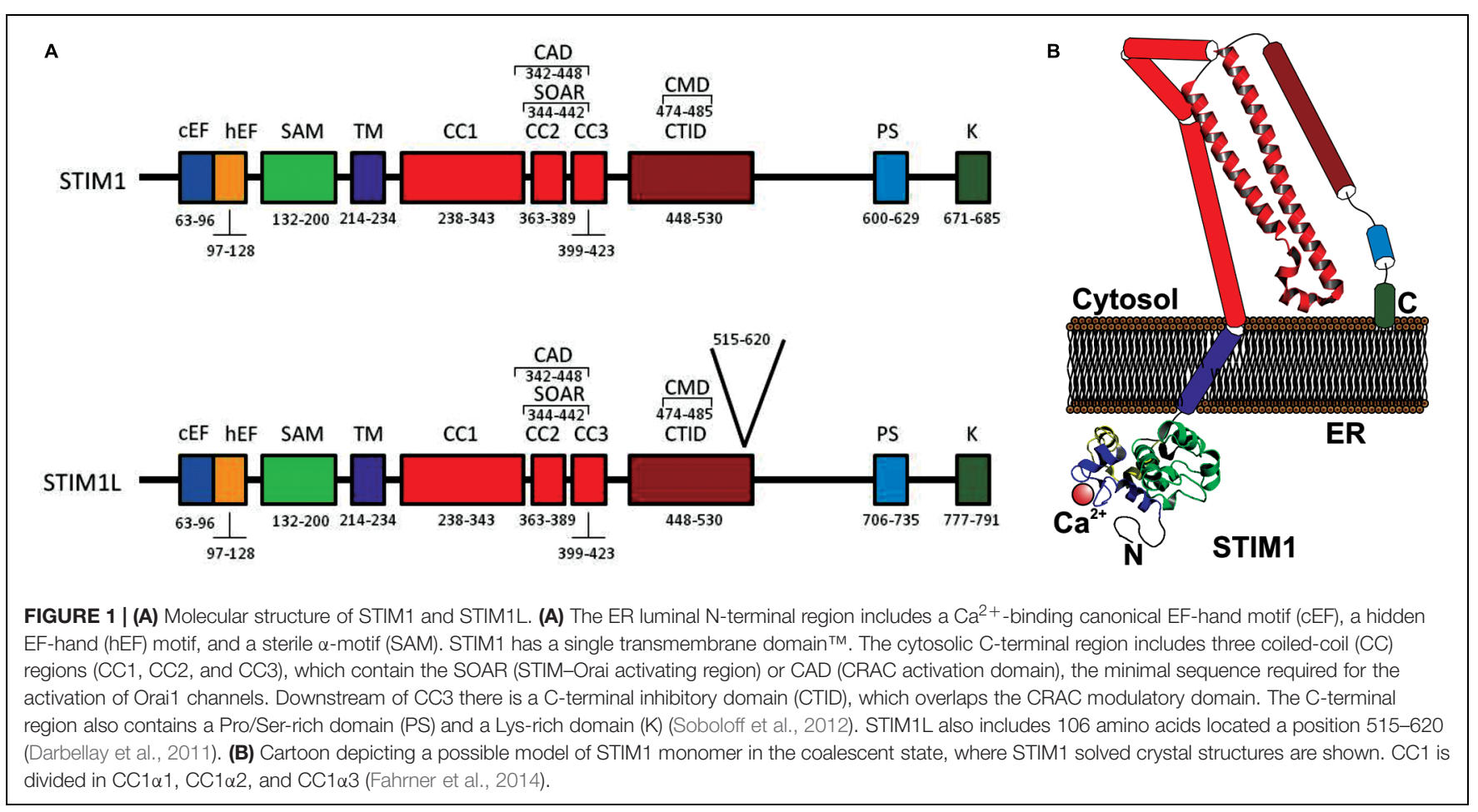

within the N-terminus (Derler et al., 2013) which contributes to STIM1 binding. Briefly, the pore acts as a funnel formed by the external vestibule, negatively charged (aa D110, D112, and D114) and supposed to attract $\mathrm{Ca}^{2+}$ to the immediacies to the pore; next the selectivity filter (aa E106); the hydrophobic cavity (aa V102, F99 and L95) and a basic region (aa R91, K87, and R83). It is surrounded by three rings, subsequently comprised by TM2, TM3, and TM4 (Hou et al., 2012). Even though TM2-4 do not form the pore themselves, it is known that several residues within those, are key regulators of the closed stated of Orail. For instance, the Orail mutations L138F and P245L, located in TM2 and TM4, trigger constitutive currents and have been found to be causing tubular aggregate myopathy (TAM; Endo et al., 2015) and the Stormorken disease (Nesin et al., 2014), respectively. Included into the $\mathrm{C}$-terminus, and in addition to the STIM1-binding region, Orail monomers exhibit a highly conserved hinge region, which allows the Orail subunits to pair with its neighbor in an antiparallel manner, and to coexist as dimers (Hou et al., 2012).

\section{STIM1-ORAI1 COUPLING}

STIM1-mediated Orail activation has been studied since their partnership was disclosed in 2006. $\mathrm{ER} \mathrm{Ca}^{2+}$ depletion prompted by a stimulus leads to $\mathrm{Ca}^{2+}$ displacement from the STIM1 EFhand and a number of rearrangements within the $\mathrm{N}$-terminus domain, transferred through the TM domain to the C-termini, which culminates in Orail activation and $\mathrm{Ca}^{2+}$ entry. Nowadays and despite to the fact that a resting STIM1 crystal structure has proved elusive, it is common consensus that in its coalescent state, STIM1 forms dimers, its EF-hand domain is occupied by $\mathrm{Ca}^{2+}$, and that the Orail triggering regions are hidden from the channel (Fahrner et al., 2013). While STIM1 response obeys solely to large variations in $\left[\mathrm{Ca}^{2+}\right]_{\mathrm{ER}}$, STIM2 shows faster reaction to smaller changes in intraluminal $\mathrm{Ca}^{2+}$ (Stathopulos et al., 2009). As stated above, $\mathrm{Ca}^{2+}$ dissociation from the EFhand changes both the later and SAM conformation exposing hydrophobic domains and habilitating the formation of STIM1 dimers and oligomers (Stathopulos et al., 2006, 2009). Next, the TM region, which has been recently shown to interact in an angled manner within the ER membrane, provides support to the control of the active/inactive state of STIM1 dimers. Upon ER depletion the angle lessened bringing the C-termini together (Ma et al., 2015). Ultimately, the signal travels through the molecule to the cytosolic domain where $\mathrm{CC} 1$, which is clamping the rest of C-terminal portion in a tight state, releases it in order to reinforce the oligomerizated conformation, via the SHD region, and to grant STIM1 activating regions access to Orail C- and N-terminus (Derler et al., 2013; Stathopulos et al., 2013; Fahrner et al., 2014). The interaction between STIM1 C-terminus and Orail C-terminus has been recently solved by NMR. In Stathopulos et al. (2013) have demonstrated that the critical positively charged residues mentioned above, K382, K284, K385, and K386, two aromatic ones Y361 and Y362; and finally four hydrophobic amino acids L347, L351, L373, and A376 are the key players within STIM1 to interact with Orail; meanwhile the channel includes the residues L273, L276, R281, L286, R289 from its C-terminus, forming what the authors have named the STIM1-Orail Association Pocket (SOAP; Stathopulos et al., 2013). However, the association between STIM1 and Orail N-terminus is still yet completely unsolved and further 


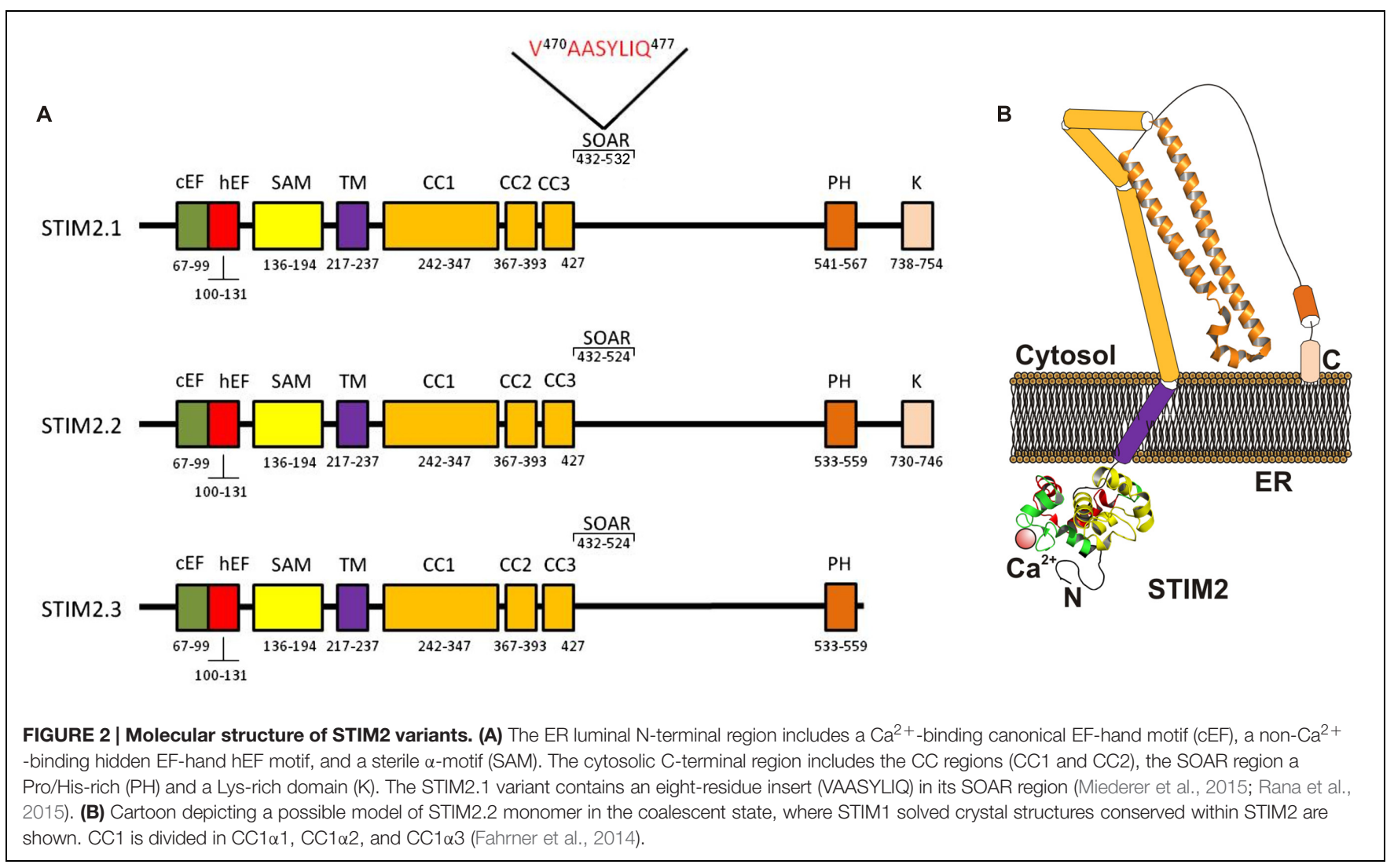

approaches are required to fully understand the STIM1-mediated gating mechanism of Orail.

Recent studies have revealed the expression of splice variants of STIM1, STIM2, and Orai1 in different cell types. It has been reported that over $95 \%$ multiexonal proteins in vertebrates undergo alternative splicing (Kornblihtt et al., 2013), which expands the functional diversity of a number of genes. Here we present the most prominent STIM1 and Orail variants and the differences among them.

\section{STIM SPLICING VARIANTS}

\section{STIM1}

As mentioned before, upon cell stimulation, the ER $\mathrm{Ca}^{2+}$ concentration decreases and $\mathrm{Ca}^{2+}$ unbinds from the canonical EF-hand, leading to an oligomerization of STIM1 molecules followed by a translocation toward the PM and aggregation as punctae structures (Lewis, 2007). Subsequently, STIM1 C-terminal region unfolds, exposing the SOAR domain and locating the polybasic lysine-rich region close to the SOC channels.

Darbellay et al. (2011) an alternatively spliced long variant of STIM1 (STIM1L, L for long to differentiate it from the conventional STIM1 isofom of $90 \mathrm{kDa}$ ) was identified in adult human muscle fibers and in in vitro-differentiated myotubes. STIM1L was described to be the product of an alternative splicing on exon 11, and contains an extra 106 residues (aa
515-620) inserted in the cytosolic region (Figure 1), an actinbinding domain, that allows STIM1L to interact with Orail $\mathrm{Ca}^{2+}$ channels to form permanent clusters (Darbellay et al., 2011).

While STIM1 is ubiquitously expressed, STIM1L is expressed in human skeletal muscle (Horinouchi et al., 2012), in skeletal muscle, as well as in heart and brain of mice (Darbellay et al., 2011) and in neonatal rat cardiomyocytes (Luo et al., 2012). The expression of STIM1L, as well as that of the conventional STIM1 variant, decreased in adult rat cardiomyocytes, where their expression levels have been found to be upregulated under pathological cardiac hypertrophy (Luo et al., 2012).

The function of STIM1L has been associated to the particularly rapid maximal activation of SOCE in skeletal muscle cells $(<1 \mathrm{~s})$ in comparison with other cells where full SOCE activation requires several seconds ( $>5 \mathrm{~s}$ in human platelets (Redondo et al., 2006), and up to $260 \mathrm{~s}$ in other cell types (Parekh and Putney, 2005; Liou et al., 2007). STIM1L was initially found to allow rapid activation of SOCE and is required to trigger repetitive cytosolic $\mathrm{Ca}^{2+}$ signals (Darbellay et al., 2011). The rapid activation of SOCE in STIM1L expressing cells was proposed to rely on the interaction between STIM1L and Orail at rest even when $\mathrm{Ca}^{2+}$ stores were full. This interaction was suggested to be stabilized by STIM1L-actin filament association as actin depolymerization has been found to disrupt STIM1L-Orail complexes at rest, which, subsequently delays SOCE activation (Darbellay et al., 2011). More recent studies have revealed that both STIM1 and STIM1L are distributed throughout the cortical ER vesicles, while Orai1 channels are localized in the PM. Following agonist stimulation 


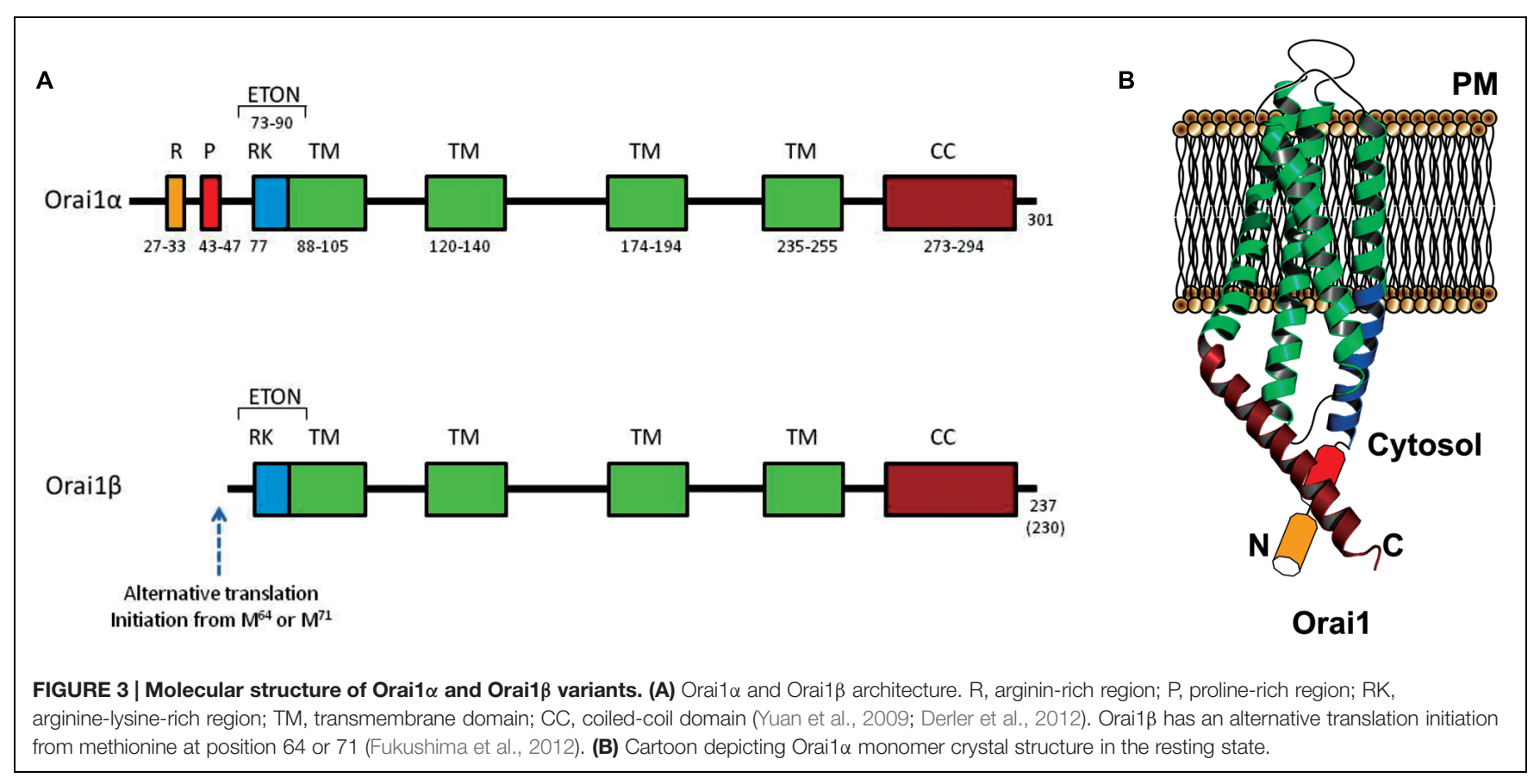

and reduction in $\mathrm{ER} \mathrm{Ca}^{2+}$ concentration STIM1 induces cortical ER expansion by a mechanism that requires the lysine-rich motif thus recruiting Orail channels in large ER-PM clusters. By contrast, STIM1L is unable to enlarge cortical ER structures and recruits Orail channels in reduced ER-PM clusters (Sauc et al., 2015). The greater efficiency of STIM1L mediating $\mathrm{Ca}^{2+}$ entry through SOCE has been hypothesized to occur due to the slower diffusion of $\mathrm{Ca}^{2+}$ in the cytoplasm in large STIM1Orail clusters which might trap $\mathrm{Ca}^{2+}$ in the proximity of Orail channels promoting $\mathrm{Ca}^{2+}$-dependent inactivation of the channel (Sauc et al., 2015).

Furthermore, STIM1L has been reported to modulate store-independent $\mathrm{Ca}^{2+}$ entry through the TRPC channels TRPC3 and TRPC6. In HEK-293 cells stably expressing endothelin type A receptor STIM1L expression was found to attenuate receptor-operated $\mathrm{Ca}^{2+}$ entry via TRPC3 and TRPC6 more strongly that STIM1 by interaction with both channels (Horinouchi et al., 2012). Overexpression of STIM1 and STIM1L did not modify the expression level of TRPC3 and TRPC6. Although STIM1L exhibits a greater capacity to bind TRPC3 and TRPC6 than STIM1 (Horinouchi et al., 2012), which might suggest the recruitment of these channels into the store-dependent signalplex, the precise mechanism involved in the suppression of storeindependent, receptor-operated, $\mathrm{Ca}^{2+}$ entry by STIM1 and STIM1L in this cell model has not been further clarified.

\section{STIM2}

The homologue of the STIM1 protein, STIM2 was identified in Williams et al. (2001) as a type I TM protein located in the ER and also identified in acidic organelles (Liou et al., 2005; Zbidi et al., 2011). The human STIM2 gene comprises 13 exons located at 4p15.1 (Williams et al., 2001), which lead to a variety of splice isoforms with different properties.

The best characterized STIM2 isoform is STIM2.2 (Miederer et al., 2015), also known as STIM2 $\alpha$ (Rana et al., 2015). Human STIM2.2 consists of 833 amino acid with a molecular weight of 105 (115 kDa for the phosphorylated form), which shares amino acid sequence as well as domain architecture with STIM1 (Lopez et al., 2012). STIM2.2 mRNA is encoded by 12 exons (exons 1-8 and 10-13).

As previously mentioned for STIM1, the N-terminal region of STIM2 is located in the lumen of the ER and comprises a canonical EF-hand motif, a "hidden" EF-hand motif, and a SAM, (Soboloff et al., 2006). The canonical EF-hand motif has the ability to bind $\mathrm{Ca}^{2+}$ and exhibits an affinity for $\mathrm{Ca}^{2+}$ greater than that of STIM1 (STIM2 EF-hand motif Kd $\sim 0.5 \mathrm{mM}$, STIM1 EF-hand motif Kd 0.6 mM; Stathopulos et al., 2006; Zheng et al., 2008), therefore, STIM2 protein shows a greater sensitivity to minor changes in $\mathrm{ER} \mathrm{Ca}^{2+}$ concentration as compared to STIM1. As a result, STIM2 has been reported to be partially active at resting ER $\mathrm{Ca}^{2+}$ concentrations and further actives by small reductions in ER $\mathrm{Ca}^{2+}$ concentrations, while STIM1 requires much larger reductions in $\mathrm{ER} \mathrm{Ca}^{2+}$ concentration, such as those induced by physiological agonists, to become active (Brandman et al., 2007). Such greater sensitivity for free $\mathrm{Ca}^{2+}$ confers STIM2 the ability to sense ER $\mathrm{Ca}^{2+}$ concentration fluctuations as well as to activate earlier than STIM1 upon agonist-induced $\mathrm{ER} \mathrm{Ca}^{2+}$ store discharge (Brandman et al., 2007).

The hEF domain, which is unable to bind $\mathrm{Ca}^{2+}$, plays an important role in the stability of the canonical EF-hand motif and SAM domains (Stathopulos et al., 2006, 2008; Zheng et al., 2011). On the other hand, the SAM domain plays an essential role in STIM oligomerization (Zheng et al., 2008, 2011). 
The C-terminal region of STIM2 is located in the cytosol and comprises an ezrin/radixin/moesin (ERM) domain that contains three CC domains (CC1-3) including the including the SOAR/CAD, which has been reported to bind to Orai and TRPC channels (Yuan et al., 2009; Lee et al., 2014; Prakriya and Lewis, 2015). STIM2 structure differs from STIM1 in the adjacent proline- and histidine-rich $(\mathrm{P} / \mathrm{H})$ motif (PHAPHPSHPRHPHHPQHTPHSLPSPDP) located in a position that resembles that of the serine- and proline-rich $(\mathrm{S} / \mathrm{P})$ region present in STIM1 (SPSAPPGGSPHLDSSRSHSPSSPDPDTPSP), whose function is still unclear. From this point the sequences of STIM1 and STIM2 are significantly different, except for the distal lysine-rich (K) motif which consists of 14 amino acids in STIM1 (five lysines) and 17 residues in STIM2 (nine lysines; Williams et al., 2001).

The function of STIM2 differs from that of STIM1. While STIM1 is the main activator of SOCE, STIM2 mainly controls the resting cytosolic and $\mathrm{ER} \mathrm{Ca}^{2+}$ concentrations and modulates prolonged $\mathrm{Ca}^{2+}$ entry and response to low concentrations of physiological agonists (Brandman et al., 2007; Oh-Hora et al., 2008). STIM2 is also involved in the regulation of the storeoperated $I_{\min } \mathrm{Ca}^{2+}$ channels in HEK-293 cells (Shalygin et al., 2015). In addition, the greater sensitivity of STIM2 to changes in $\mathrm{ER} \mathrm{Ca}^{2+}$ concentration mentioned above leads to a role for STIM2 in the regulation of $\mathrm{Ca}^{2+}$ oscillations that differs from that attributed to STIM1. Thus, silencing of STIM2 expression has been reported to impair agonist-mediated $\mathrm{Ca}^{2+}$ oscillations at low levels of store depletion, without interfering with STIM1mediated $\mathrm{Ca}^{2+}$ responses induced by full store discharge (Thiel et al., 2013).

Although the role of STIM2 in the activation of SOCE and its interaction with store-operated channels has been less investigated than that of STIM1, it has been reported that STIM2 can interact functionally with overexpressed as well as endogenously expressed Orai1, -2 , and -3 and TRPC1 (Brandman et al., 2007; Parvez et al., 2008; Bandyopadhyay et al., 2011; Zbidi et al., 2011; Berna-Erro et al., 2012; Kar et al., 2012; Stanisz et al., 2014); however, whether STIM2 is relevant for the activation of store-independent $\mathrm{Ca}^{2+}$ entry remains unclear.

Two recent studies have identified three STIM2 splice variants: STIM2.1, STIM2.2, and STIM2.3 (Miederer et al., 2015; Rana et al., 2015). The structure and function described above for STIM2 concerns the STIM2.2 variant (Figure 2), the conventional isoform of STIM2, which will be named STIM2.2 from now on.

STIM2.1, also known as STIM2 $\beta$, has been reported to contain an eight-residue insert (VAASYLIQ) in its SOAR/CAD region, encoded by an additional exon 9, that disrupts binding to Orai (Miederer et al., 2015; Rana et al., 2015; Figure 2). The expression of the STIM2.1 variant has been reported to be ubiquitous and its abundance relative to STIM2.2 depends upon the cell type but is significantly high in naive $\mathrm{T}$ cells, where the expression of both variants is similar (Miederer et al., 2015). In contrast to the role of STIM2.2 as an activator of SOCE, STIM2.1 has been shown to play an inhibitory role. STIM2.1 knockdown increases SOCE in $\mathrm{CD}^{+} \mathrm{T}$ cells, while overexpression of STIM2.1 decreases SOCE (Miederer et al., 2015).
The mechanism underlying the inhibitory role of the STIM2.1 variant in SOCE remains unclear. STIM2.1 by itself has been reported to interact poorly with Orail as detected by FRET or puncta formation assays (Rana et al., 2015); however, it has been indicated that STIM2.1 might heterodimerize with STIM1 or STIM2.2, which might recruit it to Orail channels, increasing the possibility to inhibit SOCE despite its low affinity for the channel. When STIM2.1 is recruited to the Orail channel signalplex, it might inhibit SOCE passively by direct interaction with STIM1 or STIM2.2, thus reducing the number of SOAR/CAD domains available for channel activation. However, this passive inhibition is unlikely to play a significant role in the modulation of SOCE under physiological conditions, since STIM2.1 is generally not as highly expressed as STIM1, and, therefore, a stronger active inhibitory role has also been hypothesized (Rana et al., 2015).

The transcript of the third STIM2 variant, STIM2.3, contains an alternative exon 13 that leads to an upstream end of translation and a transcript shortened by $444 \mathrm{bp}$, which results in a protein with approximately $17 \mathrm{kDa}$ smaller (Miederer et al., 2015; Figure 2). The function of the STIM2.3 variant is unknown at present and its expression seems to be quite limited and has not been detected in lymphocytes, where the other two variants are significantly expressed (Miederer et al., 2015).

\section{ORAI1 SPLICING VARIANTS}

Two variants of Orai1, Orail $\alpha$, and Orai $1 \beta$, have been found to be expressed. Orail $\alpha$ is the conventional variant of 301 amino acids ( $\sim 33 \mathrm{kDa}$, although the predicted molecular weight might be significantly modified by post-translational modifications, such as glycosylation on the asparagine residue at position 223 (Gwack et al., 2007) or phosphorylation on serine residues at positions 27 and 30 (Kawasaki et al., 2010). The short Orail variant, Orai1 $\beta$, is generated by alternative translation initiation from a methionine at position 64 , and possibly also 71 , leading to a protein of approximately $23 \mathrm{kDa}$. Both Orail variants have been found to be ubiquitously expressed in human cell lines from a number of tissues, including HEK293 cells, Jurkat T cells, HeLa cells, epidermal HaCaT cells or the T84 lung carcinoma cell line, and show similar cellular localization (Fukushima et al., 2012).

The sequence upstream of the translation initiation of Orai1 $\beta$ includes a proline-rich motif previously suggested to be important for Orail gating by STIM1 (Takahashi et al., 2007), and an arginine-rich sequence that has been found to be involved in the interaction of Orail with PM phosphatidylinositol4,5-bisphosphate that might be important for the mobility of Orail in the PM (Calloway et al., 2011). In agreement with the latter, Fukushima and coworkers have reported that Orai1 $\beta$ has faster mobility in the PM (Fukushima et al., 2012).

A recent report by Desai et al. (2015) has revealed that both Orail variants might be subunits of the store-operated CRAC and SOC channels, with some biophysical differences that includes a stronger $\mathrm{Ca}^{2+}$-dependent inactivation of Orail $\alpha$. However, 
the most significant functional difference between these variants lies in the participation of Orail $\alpha$, but not Orail $\beta$, in the ARC channels (Desai et al., 2015), although the molecular mechanisms underlying the different biological significance of both variants remain unclear.

Summarizing, different STIM1, STIM2, and Orai1 variants have been reported to be expressed as a result of alternative splicing in a number of cell types. The expression of certain variants, such as STIM1L, is quite restricted while that of other variants is ubiquitous. As it has been hypothesized (Kornblihtt et al., 2013), alternative splicing might expands the functional diversity of multiexonal genes. Consistent with this, significant functional differences have been reported between STIM1 and STIM1L, STIM2.1 and STIM2.2 and between the $\alpha$ and $\beta$ variants of Orai1, which lead to distinct mechanisms of regulation of $\mathrm{Ca}^{2+}$ entry through store-operated (CRAC and SOC) as well as storeindependent (ARC) channels. The analysis of the expression

\section{REFERENCES}

Baba, Y., Hayashi, K., Fujii, Y., Mizushima, A., Watarai, H., Wakamori, M., et al. (2006). Coupling of STIM1 to store-operated Ca2+ entry through its constitutive and inducible movement in the endoplasmic reticulum. Proc. Natl. Acad. Sci. U.S.A. 103, 16704-16709. doi: 10.1073/pnas.0608358103

Bandyopadhyay, B. C., Pingle, S. C., and Ahern, G. P. (2011). Store-operated $\mathrm{Ca}(2)+$ signaling in dendritic cells occurs independently of STIM1. J. Leukoc. Biol. 89, 57-62. doi: 10.1189/jlb.0610381

Berna-Erro, A., Galan, C., Dionisio, N., Gomez, L. J., Salido, G. M., and Rosado, J. A. (2012). Capacitative and non-capacitative signaling complexes in human platelets. Biochim. Biophys. Acta 1823, 1242-1251. doi: 10.1016/j.bbamcr.2012.05.023

Berridge, M. J., Bootman, M. D., and Roderick, H. L. (2003). Calcium signalling: dynamics, homeostasis and remodelling. Nat. Rev. Mol. Cell Biol. 4, 517-529. doi: $10.1038 / \mathrm{nrm} 1155$

Brandman, O., Liou, J., Park, W. S., and Meyer, T. (2007). STIM2 is a feedback regulator that stabilizes basal cytosolic and endoplasmic reticulum $\mathrm{Ca} 2+$ levels. Cell 131, 1327-1339. doi: 10.1016/j.cell.2007.11.039

Cahalan, M. D. (2009). STIMulating store-operated Ca(2+) entry. Nat. Cell Biol. 11, 669-677. doi: 10.1038/ncb0609-669

Calloway, N., Owens, T., Corwith, K., Rodgers, W., Holowka, D., and Baird, B. (2011). Stimulated association of STIM1 and Orail is regulated by the balance of PtdIns(4,5)P(2) between distinct membrane pools. J. Cell Sci. 124, 2602-2610. doi: $10.1242 /$ jcs.084178

Cheng, K. T., Ong, H. L., Liu, X., and Ambudkar, I. S. (2011). Contribution of TRPC1 and Orail to $\mathrm{Ca}(2+)$ entry activated by store depletion. Adv. Exp. Med. Biol. 704, 435-449. doi: 10.1007/978-94-007-0265-3_24

Cheng, K. T., Ong, H. L., Liu, X., and Ambudkar, I. S. (2013). Contribution and regulation of TRPC channels in store-operated Ca2+ entry. Curr. Top. Membr. 71, 149-179. doi: 10.1016/B978-0-12-407870-3.00007-X

Choi, S., Maleth, J., Jha, A., Lee, K. P., Kim, M. S., So, I., et al. (2014). The TRPCs-STIM1-orai interaction. Handb. Exp. Pharmacol. 223, 1035-1054. doi: 10.1007/978-3-319-05161-1_13

Covington, E. D., Wu, M. M., and Lewis, R. S. (2010). Essential role for the CRAC activation domain in store-dependent oligomerization of STIM1. Mol. Biol. Cell 21, 1897-1907. doi: 10.1091/mbc.E10-02-0145

Darbellay, B., Arnaudeau, S., Bader, C. R., Konig, S., and Bernheim, L. (2011). STIM1L is a new actin-binding splice variant involved in fast repetitive $\mathrm{Ca} 2+$ release. J. Cell Biol. 194, 335-346. doi: 10.1083/jcb.201 012157

Derler, I., Madl, J., Schutz, G., and Romanin, C. (2012). Structure, regulation and biophysics of I(CRAC), STIM/Orail. Adv. Exp. Med. Biol. 740, 383-410. doi: 10.1007/978-94-007-2888-2_16

Derler, I., Plenk, P., Fahrner, M., Muik, M., Jardin, I., Schindl, R., et al. (2013). The extended transmembrane Orail N-terminal (ETON) region combines ratios of the different variants in a particular cellular model might be of great interest to understand the fine modulation of $\mathrm{Ca}^{2+}$ entry.

\section{AUTHOR CONTRIBUTIONS}

JR designed the manuscript, contribute to write it and performed the final edition. $\mathrm{RD}$, TS, and IJ contributed to write the manuscript and discussion.

\section{ACKNOWLEDGMENTS}

This work was supported by MINECO (Grants BFU2013-45564C2-1-P and BFU2013-45564-C2-2-P) and Junta de ExtremaduraFEDER (GR15029).

binding interface and gate for Orail activation by STIM1. J. Biol. Chem. 288, 29025-29034. doi: 10.1074/jbc.M113.501510

Desai, P. N., Zhang, X., Wu, S., Janoshazi, A., Bolimuntha, S., Putney, J. W., et al. (2015). Multiple types of calcium channels arising from alternative translation initiation of the Orail message. Sci. Signal. 8, ra74. doi: 10.1126/scisignal.aaa8323

Endo, Y., Noguchi, S., Hara, Y., Hayashi, Y. K., Motomura, K., Miyatake, S., et al. (2015). Dominant mutations in ORAIl cause tubular aggregate myopathy with hypocalcemia via constitutive activation of store-operated $\mathrm{Ca}(2)(+)$ channels. Hum. Mol. Genet. 24, 637-648. doi: 10.1093/hmg/ddu477

Fahrner, M., Derler, I., Jardin, I., and Romanin, C. (2013). The STIM1/Orai signaling machinery. Channels (Austin) 7, 330-343. doi: 10.4161/chan.26742

Fahrner, M., Muik, M., Schindl, R., Butorac, C., Stathopulos, P., Zheng, L., et al. (2014). A coiled-coil clamp controls both conformation and clustering of stromal interaction molecule 1 (STIM1). J. Biol. Chem. 289, 33231-33244. doi: 10.1074/jbc.M114.610022

Feske, S., Gwack, Y., Prakriya, M., Srikanth, S., Puppel, S. H., Tanasa, B., et al. (2006). A mutation in Orail causes immune deficiency by abrogating CRAC channel function. Nature 441, 179-185. doi: 10.1038/nature04702

Feske, S., Prakriya, M., Rao, A., and Lewis, R. S. (2005). A severe defect in CRAC $\mathrm{Ca} 2+$ channel activation and altered $\mathrm{K}^{+}$channel gating in $\mathrm{T}$ cells from immunodeficient patients. J. Exp. Med. 202, 651-662. doi: 10.1084/jem.20050687

Fukushima, M., Tomita, T., Janoshazi, A., and Putney, J. W. (2012). Alternative translation initiation gives rise to two isoforms of Orail with distinct plasma membrane mobilities. J. Cell Sci. 125, 4354-4361. doi: 10.1242/jcs.104919

Gwack, Y., Srikanth, S., Feske, S., Cruz-Guilloty, F., Oh-Hora, M., Neems, D. S., et al. (2007). Biochemical and functional characterization of Orai proteins. J. Biol. Chem. 282, 16232-16243. doi: 10.1074/jbc.M609630200

Horinouchi, T., Higashi, T., Higa, T., Terada, K., Mai, Y., Aoyagi, H., et al. (2012). Different binding property of STIM1 and its novel splice variant STIM1L to Orai1, TRPC3, and TRPC6 channels. Biochem. Biophys. Res. Commun. 428, 252-258. doi: 10.1016/j.bbrc.2012.10.034

Hou, X., Pedi, L., Diver, M. M., and Long, S. B. (2012). Crystal structure of the calcium release-activated calcium channel Orai. Science 338, 1308-1313. doi: $10.1126 /$ science. 1228757

Huang, G. N., Zeng, W., Kim, J. Y., Yuan, J. P., Han, L., Muallem, S., et al. (2006). STIM1 carboxyl-terminus activates native SOC, I(crac) and TRPC1 channels. Nat. Cell Biol. 8, 1003-1010. doi: 10.1038/ncb1454

Jardin, I., Dionisio, N., Frischauf, I., Berna-Erro, A., Woodard, G. E., Lopez, J. J., et al. (2013). The polybasic lysine-rich domain of plasma membraneresident STIM1 is essential for the modulation of store-operated divalent cation entry by extracellular calcium. Cell. Signal. 25, 1328-1337. doi: 10.1016/j.cellsig.2013.01.025

Jardin, I., Lopez, J. J., Redondo, P. C., Salido, G. M., and Rosado, J. A. (2009). Store-operated $\mathrm{Ca} 2+$ entry is sensitive to the extracellular $\mathrm{Ca} 2+$ concentration 
through plasma membrane STIM1. Biochim. Biophys. Acta 1793, 1614-1622. doi: 10.1016/j.bbamcr.2009.07.003

Jardin, I., Lopez, J. J., Salido, G. M., and Rosado, J. A. (2008). Orail mediates the interaction between STIM1 and hTRPC1 and regulates the mode of activation of hTRPC1-forming Ca2+ channels. J. Biol. Chem. 283, 25296-25304. doi: 10.1074/jbc.M802904200

Kar, P., Bakowski, D., Di Capite, J., Nelson, C., and Parekh, A. B. (2012). Different agonists recruit different stromal interaction molecule proteins to support cytoplasmic $\mathrm{Ca} 2+$ oscillations and gene expression. Proc. Natl. Acad. Sci. U.S.A. 109, 6969-6974. doi: 10.1073/pnas.1201204109

Kawasaki, T., Lange, I., and Feske, S. (2009). A minimal regulatory domain in the $\mathrm{C}$ terminus of STIM1 binds to and activates ORAI1 CRAC channels. Biochem. Biophys. Res. Commun. 385, 49-54. doi: 10.1016/j.bbrc.2009. 05.020

Kawasaki, T., Ueyama, T., Lange, I., Feske, S., and Saito, N. (2010). Protein kinase $\mathrm{C}$-induced phosphorylation of Orail regulates the intracellular $\mathrm{Ca} 2+$ level via the store-operated Ca2+ channel. J. Biol. Chem. 285, 25720-25730. doi: 10.1074/jbc.M109.022996

Kornblihtt, A. R., Schor, I. E., Allo, M., Dujardin, G., Petrillo, E., and Munoz, M. J. (2013). Alternative splicing: a pivotal step between eukaryotic transcription and translation. Nat. Rev. Mol. Cell Biol. 14, 153-165. doi: 10.1038/nrm3525

Lee, K. P., Choi, S., Hong, J. H., Ahuja, M., Graham, S., Ma, R., et al. (2014). Molecular determinants mediating gating of Transient Receptor Potential Canonical (TRPC) channels by stromal interaction molecule 1 (STIM1). J. Biol. Chem. 289, 6372-6382. doi: 10.1074/jbc.M113.546556

Lewis, R. S. (2007). The molecular choreography of a store-operated calcium channel. Nature 446, 284-287. doi: 10.1038/nature05637

Liou, J., Fivaz, M., Inoue, T., and Meyer, T. (2007). Live-cell imaging reveals sequential oligomerization and local plasma membrane targeting of stromal interaction molecule 1 after $\mathrm{Ca} 2+$ store depletion. Proc. Natl. Acad. Sci. U.S.A. 104, 9301-9306. doi: 10.1073/pnas.0702866104

Liou, J., Kim, M. L., Heo, W. D., Jones, J. T., Myers, J. W., Ferrell, J. E., et al. (2005). STIM is a Ca2+ sensor essential for $\mathrm{Ca} 2+$-store-depletion-triggered Ca2+ influx. Curr. Biol. 15, 1235-1241. doi: 10.1016/j.cub.2005.05.055

Lopez, E., Salido, G. M., Rosado, J. A., and Berna-Erro, A. (2012). Unraveling STIM2 function. J. Physiol. Biochem. 68, 619-633. doi: 10.1007/s13105-0120163-1

Luik, R. M., Wang, B., Prakriya, M., Wu, M. M., and Lewis, R. S. (2008). Oligomerization of STIM1 couples ER calcium depletion to CRAC channel activation. Nature 454, 538-542. doi: 10.1038/nature07065

Luo, X., Hojayev, B., Jiang, N., Wang, Z. V., Tandan, S., Rakalin, A., et al. (2012). STIM1-dependent store-operated $\mathrm{Ca}(2)(+)$ entry is required for pathological cardiac hypertrophy. J. Mol. Cell. Cardiol. 52, 136-147. doi: 10.1016/j.yjmcc.2011.11.003

Ma, G., Wei, M., He, L., Liu, C., Wu, B., Zhang, S. L., et al. (2015). Inside-out $\mathrm{Ca}(2+)$ signalling prompted by STIM1 conformational switch. Nat. Commun. 6, 7826. doi: $10.1038 /$ ncomms 8826

Maruyama, Y., Ogura, T., Mio, K., Kato, K., Kaneko, T., Kiyonaka, S., et al. (2009). Tetrameric Orail is a teardrop-shaped molecule with a long, tapered cytoplasmic domain. J. Biol. Chem. 284, 13676-13685. doi: 10.1074/jbc.M900812200

Mercer, J. C., Dehaven, W. I., Smyth, J. T., Wedel, B., Boyles, R. R., Bird, G. S., et al. (2006). Large store-operated calcium selective currents due to co-expression of Orail or Orai2 with the intracellular calcium sensor, Stim1. J. Biol. Chem. 281, 24979-24990. doi: 10.1074/jbc.M604589200

Miederer, A. M., Alansary, D., Schwar, G., Lee, P. H., Jung, M., Helms, V., et al. (2015). A STIM2 splice variant negatively regulates store-operated calcium entry. Nat. Commun. 6, 6899. doi: 10.1038/ncomms 7899

Mignen, O., Thompson, J. L., and Shuttleworth, T. J. (2007). STIM1 regulates Ca2+ entry via arachidonate-regulated $\mathrm{Ca} 2+$-selective (ARC) channels without storedepletion or translocation to the plasma membrane. J. Physiol. (Lond.) $579(\mathrm{Pt}$ 3), 703-715. doi: 10.1113/jphysiol.2006.122432

Mignen, O., Thompson, J. L., and Shuttleworth, T. J. (2008a). Both Orail and Orai3 are essential components of the arachidonate-regulated $\mathrm{Ca}_{2}{ }^{-}$-selective (ARC) channels. J. Physiol. 586, 185-195. doi: 10.1113/jphysiol.2007.146258

Mignen, O., Thompson, J. L., and Shuttleworth, T. J. (2008b). Orail subunit stoichiometry of the mammalian CRAC channel pore. J. Physiol. 586, 419-425. doi: 10.1113/jphysiol.2007.147249
Mignen, O., Thompson, J. L., and Shuttleworth, T. J. (2009). The molecular architecture of the arachidonate-regulated Ca2+-selective ARC channel is a pentameric assembly of Orai1 and Orai3 subunits. J. Physiol. 587, 4181-4197. doi: $10.1113 /$ jphysiol.2009.174193

Muik, M., Fahrner, M., Derler, I., Schindl, R., Bergsmann, J., Frischauf, I., et al. (2009). A cytosolic homomerization and a modulatory domain within STIM1 C terminus determine coupling to ORAI1 channels. J. Biol. Chem. 284, 8421-8426. doi: 10.1074/jbc.C800229200

Nesin, V., Wiley, G., Kousi, M., Ong, E. C., Lehmann, T., Nicholl, D. J., et al. (2014). Activating mutations in STIM1 and ORAI1 cause overlapping syndromes of tubular myopathy and congenital miosis. Proc. Natl. Acad. Sci. U.S.A. 111, 4197-4202. doi: 10.1073/pnas.1312520111

Oh-Hora, M., Yamashita, M., Hogan, P. G., Sharma, S., Lamperti, E., Chung, W., et al. (2008). Dual functions for the endoplasmic reticulum calcium sensors STIM1 and STIM2 in T cell activation and tolerance. Nat. Immunol. 9, 432-443. doi: $10.1038 /$ ni1574

Oritani, K., and Kincade, P. W. (1996). Identification of stromal cell products that interact with pre-B cells. J. Cell Biol. 134, 771-782. doi: 10.1083/jcb. 134.3.771

Palty, R., and Isacoff, E. Y. (2016). Cooperative binding of stromal interaction molecule 1 (STIM1) to the $\mathrm{N}$ and $\mathrm{C}$ termini of calcium release-activated calcium modulator 1 (Orai1). J. Biol. Chem. 291, 334-341. doi: 10.1074/jbc.M115.685289

Palty, R., Stanley, C., and Isacoff, E. Y. (2015). Critical role for Orail C-terminal domain and TM4 in CRAC channel gating. Cell Res. 25, 963-980. doi: $10.1038 /$ cr.2015.80

Parekh, A. B., and Putney, J. W. Jr (2005). Store-operated calcium channels. Physiol. Rev. 85, 757-810. doi: 10.1152/physrev.00057.2003

Park, C. Y., Hoover, P. J., Mullins, F. M., Bachhawat, P., Covington, E. D., Raunser, S., et al. (2009). STIM1 clusters and activates CRAC channels via direct binding of a cytosolic domain to Orai1. Cell 136, 876-890. doi: 10.1016/j.cell.2009.02.014

Parvez, S., Beck, A., Peinelt, C., Soboloff, J., Lis, A., Monteilh-Zoller, M., et al. (2008). STIM2 protein mediates distinct store-dependent and storeindependent modes of CRAC channel activation. FASEB J. 22, 752-761. doi: 10.1096/fj.07-9449com

Peinelt, C., Vig, M., Koomoa, D. L., Beck, A., Nadler, M. J., Koblan-Huberson, M., et al. (2006). Amplification of CRAC current by STIM1 and CRACM1 (Orai1). Nat. Cell Biol. 8, 771-773. doi: 10.1038/ncb1435

Penna, A., Demuro, A., Yeromin, A. V., Zhang, S. L., Safrina, O., Parker, I., et al. (2008). The CRAC channel consists of a tetramer formed by Stim-induced dimerization of Orai dimers. Nature 456, 116-120. doi: 10.1038/nature07338

Prakriya, M., Feske, S., Gwack, Y., Srikanth, S., Rao, A., and Hogan, P. G. (2006). Orail is an essential pore subunit of the CRAC channel. Nature 443, 230-233. doi: 10.1038/nature 05122

Prakriya, M., and Lewis, R. S. (2015). Store-operated calcium channels. Physiol. Rev. 95, 1383-1436. doi: 10.1152/physrev.00020.2014

Putney, J. W. Jr. (1986). A model for receptor-regulated calcium entry. Cell Calcium 7, 1-12. doi: 10.1016/0143-4160(86)90026-6

Rana, A., Yen, M., Sadaghiani, A. M., Malmersjo, S., Park, C. Y., Dolmetsch, R. E., et al. (2015). Alternative splicing converts STIM2 from an activator to an inhibitor of store-operated calcium channels. J. Cell Biol. 209, 653-669. doi: $10.1083 /$ jcb. 201412060

Redondo, P. C., Harper, M. T., Rosado, J. A., and Sage, S. O. (2006). A role for cofilin in the activation of store-operated calcium entry by de novo conformational coupling in human platelets. Blood 107, 973-979. doi: 10.1182/blood-2005-052015

Redondo, P. C., and Rosado, J. A. (2015). Store-operated calcium entry: unveiling the calcium handling signalplex. Int. Rev. Cell Mol. Biol. 316, 183-226. doi: 10.1016/bs.ircmb.2015.01.007

Rodriguez-Moyano, M., Diaz, I., Dionisio, N., Zhang, X., Avila-Medina, J., Calderon-Sanchez, E., et al. (2013). Urotensin-II promotes vascular smooth muscle cell proliferation through store operated calcium entry and EGFR transactivation. Cardiovasc. Res. 100, 297-306. doi: 10.1093/cvr/cvt196

Roos, J., Digregorio, P. J., Yeromin, A. V., Ohlsen, K., Lioudyno, M., Zhang, S., et al. (2005). STIM1, an essential and conserved component of store-operated Ca2+ channel function. J. Cell Biol. 169, 435-445. doi: 10.1083/jcb.200502019

Rosado, J. A., and Sage, S. O. (2000). Coupling between inositol 1,4,5trisphosphate receptors and human transient receptor potential channel 1 when 
intracellular Ca2 + stores are depleted. Biochem. J. 350(Pt 3), 631-635. doi: 10.1042/bj3500631

Rothberg, B. S., Wang, Y., and Gill, D. L. (2013). Orai channel pore properties and gating by STIM: implications from the Orai crystal structure. Sci. Signal. 6, e9. doi: 10.1126/scisignal.2003971

Sabbioni, S., Veronese, A., Trubia, M., Taramelli, R., Barbanti-Brodano, G., Croce, C. M., et al. (1999). Exon structure and promoter identification of STIM1 (alias GOK), a human gene causing growth arrest of the human tumor cell lines G401 and RD. Cytogenet. Cell Genet. 86, 214-218. doi: 10.1159/000015341

Sauc, S., Bulla, M., Nunes, P., Orci, L., Marchetti, A., Antigny, F., et al. (2015). STIM1L traps and gates Orail channels without remodeling the cortical ER. J. Cell Sci. 128, 1568-1579. doi: 10.1242/jcs.164228

Shalygin, A., Skopin, A., Kalinina, V., Zimina, O., Glushankova, L., Mozhayeva, G. N., et al. (2015). STIM1 and STIM2 proteins differently regulate endogenous store-operated channels in HEK293 cells. J. Biol. Chem. 290, 4717-4727. doi: 10.1074/jbc.M114.601856

Singh, B. B., Liu, X., and Ambudkar, I. S. (2000). Expression of truncated transient receptor potential protein 1alpha (Trplalpha ): evidence that the Trp1 C terminus modulates store-operated Ca2 ${ }^{+}$entry. J. Biol. Chem. 275, 3648336486. doi: 10.1074/jbc.C000529200

Soboloff, J., Rothberg, B. S., Madesh, M., and Gill, D. L. (2012). STIM proteins: dynamic calcium signal transducers. Nat. Rev. Mol. Cell Biol. 13, 549-565. doi: $10.1038 / \mathrm{nrm} 3414$

Soboloff, J., Spassova, M. A., Hewavitharana, T., He, L. P., Xu, W., Johnstone, L. S., et al. (2006). STIM2 is an inhibitor of STIM1-mediated store-operated $\mathrm{Ca}^{+}$ Entry. Curr. Biol. 16, 1465-1470. doi: 10.1016/j.cub.2006.05.051

Spassova, M. A., Soboloff, J., He, L. P., Xu, W., Dziadek, M. A., and Gill, D. L. (2006). STIM1 has a plasma membrane role in the activation of storeoperated $\mathrm{Ca}(2+)$ channels. Proc. Natl. Acad. Sci. U.S.A. 103, 4040-4045. doi: $10.1073 /$ pnas.0510050103

Stanisz, H., Saul, S., Muller, C. S., Kappl, R., Niemeyer, B. A., Vogt, T., et al. (2014). Inverse regulation of melanoma growth and migration by Orail/STIM2dependent calcium entry. Pigment Cell Melanoma Res. 27, 442-453. doi: $10.1111 /$ pcmr. 12222

Stathopulos, P. B., Li, G. Y., Plevin, M. J., Ames, J. B., and Ikura, M. (2006). Stored $\mathrm{Ca} 2^{+}$depletion-induced oligomerization of stromal interaction molecule 1 (STIM1) via the EF-SAM region: an initiation mechanism for capacitive $\mathrm{Ca}^{+}$ entry. J. Biol. Chem. 281, 35855-35862. doi: 10.1074/jbc.M608247200

Stathopulos, P. B., Schindl, R., Fahrner, M., Zheng, L., Gasmi-Seabrook, G. M., Muik, M., et al. (2013). STIM1/Orail coiled-coil interplay in the regulation of store-operated calcium entry. Nat. Commun. 4, 2963. doi: $10.1038 /$ ncomms3963

Stathopulos, P. B., Zheng, L., and Ikura, M. (2009). Stromal interaction molecule (STIM) 1 and STIM2 calcium sensing regions exhibit distinct unfolding and oligomerization kinetics. J. Biol. Chem. 284, 728-732. doi: 10.1074/jbc.C800178200

Stathopulos, P. B., Zheng, L., Li, G. Y., Plevin, M. J., and Ikura, M. (2008). Structural and mechanistic insights into STIM1-mediated initiation of store-operated calcium entry. Cell 135, 110-122. doi: 10.1016/j.cell.2008.08.006

Takahashi, Y., Murakami, M., Watanabe, H., Hasegawa, H., Ohba, T., Munehisa, Y., et al. (2007). Essential role of the N-terminus of murine Orail in storeoperated $\mathrm{Ca}^{+}$entry. Biochem. Biophys. Res. Commun. 356, 45-52. doi: 10.1016/j.bbrc.2007.02.107

Thiel, M., Lis, A., and Penner, R. (2013). STIM2 drives Ca2 ${ }^{+}$oscillations through store-operated $\mathrm{Ca}^{+}$entry caused by mild store depletion. J. Physiol. 591, 1433-1445. doi: 10.1113/jphysiol.2012.245399

Thompson, J. L., and Shuttleworth, T. J. (2013a). How many Orai's does it take to make a CRAC channel? Nat. Sci. Rep. 3, 1961. doi: 10.1038/srep01961

Thompson, J. L., and Shuttleworth, T. J. (2013b). Molecular basis of activation of the arachidonate-regulated $\mathrm{Ca}^{+}$(ARC) channel, a store-independent
Orai channel, by plasma membrane STIM1. J. Physiol. 591, 3507-3523. doi: 10.1113/jphysiol.2013.256784

Wang, X., Wang, Y., Zhou, Y., Hendron, E., Mancarella, S., Andrake, M. D., et al. (2014). Distinct Orai-coupling domains in STIM1 and STIM2 define the Orai-activating site. Nat. Commun. 5, 3183. doi: 10.1038/ncomm s4183

Wang, Y., Deng, X., Mancarella, S., Hendron, E., Eguchi, S., Soboloff, J., et al. (2010). The calcium store sensor, STIM1, reciprocally controls Orai and CaV1.2 channels. Science 330, 105-109. doi: 10.1126/science.1191086

Williams, R. T., Manji, S. S., Parker, N. J., Hancock, M. S., Van Stekelenburg, L., Eid, J. P., et al. (2001). Identification and characterization of the STIM (stromal interaction molecule) gene family: coding for a novel class of transmembrane proteins. Biochem. J. 357, 673-685. doi: 10.1042/0264-6021:3570673

Yang, X., Jin, H., Cai, X., Li, S., and Shen, Y. (2012). Structural and mechanistic insights into the activation of Stromal interaction molecule 1 (STIM1). Proc. Natl. Acad. Sci. U.S.A. 109, 5657-5662. doi: 10.1073/pnas.1118947109

Yuan, J. P., Zeng, W., Dorwart, M. R., Choi, Y. J., Worley, P. F., and Muallem, S. (2009). SOAR and the polybasic STIM1 domains gate and regulate Orai channels. Nat. Cell Biol. 11, 337-343. doi: 10.1038/ncb1842

Yuan, J. P., Zeng, W., Huang, G. N., Worley, P. F., and Muallem, S. (2007). STIM1 heteromultimerizes TRPC channels to determine their function as store-operated channels. Nat. Cell Biol. 9, 636-645. doi: 10.1038/ncb1590

Zbidi, H., Jardin, I., Woodard, G. E., Lopez, J. J., Berna-Erro, A., Salido, G. M., et al. (2011). STIM1 and STIM2 are located in the acidic $\mathrm{Ca}_{2}{ }^{+}$stores and associates with Orail upon depletion of the acidic stores in human platelets. J. Biol. Chem. 286, 12257-12270. doi: 10.1074/jbc.M110.190694

Zeng, W., Yuan, J. P., Kim, M. S., Choi, Y. J., Huang, G. N., Worley, P. F., et al. (2008). STIM1 gates TRPC channels, but not Orai1, by electrostatic interaction. Mol. Cell 32, 439-448. doi: 10.1016/j.molcel.2008.09.020

Zhang, S. L., Yeromin, A. V., Zhang, X. H. F., Yu, Y., Safrina, O., Penna, A., et al. (2006). Genome-wide RNAi screen of $\mathrm{Ca}^{+}{ }^{+}$influx identifies genes that regulate $\mathrm{Ca} 2+$ release-activated Ca2+ channel activity. Proc. Natl. Acad. Sci. U.S.A. 103, 9357-9362. doi: 10.1073/pnas.0603161103

Zhang, S. L., Yu, Y., Roos, J., Kozak, J. A., Deerinck, T. J., Ellisman, M. H., et al. (2005). STIM1 is a Ca2 ${ }^{+}$sensor that activates CRAC channels and migrates from the $\mathrm{Ca}_{2}{ }^{+}$store to the plasma membrane. Nature 437, 902-905. doi: 10.1038/nature04147

Zhang, X., Zhang, W., Gonzalez-Cobos, J. C., Jardin, I., Romanin, C., Matrougui, K., et al. (2014). Complex role of STIM1 in the activation of store-independent Orai1/3 channels. J. Gen. Physiol. 143, 345-359. doi: 10.1085/jgp.201311084

Zheng, L., Stathopulos, P. B., Li, G. Y., and Ikura, M. (2008). Biophysical characterization of the EF-hand and SAM domain containing $\mathrm{Ca}^{+}{ }^{+}$sensory region of STIM1 and STIM2. Biochem. Biophys. Res. Commun. 369, 240-246. doi: 10.1016/j.bbrc.2007.12.129

Zheng, L., Stathopulos, P. B., Schindl, R., Li, G. Y., Romanin, C., and Ikura, M. (2011). Auto-inhibitory role of the EF-SAM domain of STIM proteins in storeoperated calcium entry. Proc. Natl. Acad. Sci. U.S.A. 108, 1337-1342. doi: $10.1073 /$ pnas. 1015125108

Conflict of Interest Statement: The authors declare that the research was conducted in the absence of any commercial or financial relationships that could be construed as a potential conflict of interest.

Copyright (C) 2016 Rosado, Diez, Smani and Jardin. This is an open-access article distributed under the terms of the Creative Commons Attribution License (CC BY). The use, distribution or reproduction in other forums is permitted, provided the original author(s) or licensor are credited and that the original publication in this journal is cited, in accordance with accepted academic practice. No use, distribution or reproduction is permitted which does not comply with these terms. 